\title{
Contribuição de grupos de pesquisa brasileiros na avaliação de fármacos para o tratamento da dependência de álcool
}

\author{
Contribution of Brazilian research groups to the evaluation of drugs for \\ alcohol dependence treatment
}

Uilton de Abreu Almeida', Ricardo Peres do Souto²

\begin{abstract}
RESUMO
Estatísticas recentes mostram que milhões de brasileiros apresentam dependência deálcool.Uma das principais estratégias para o tratamento desses dependentes é a terapia farmacológica, que ainda apresenta baixas taxas de sucesso. Nesse contexto, é de suma importância que grupos de pesquisa nacionais conduzam estudos de avaliação de fármacos para o tratamento de dependentes de álcool. Este estudo é uma revisão bibliográfica narrativa crítica realizada na base de dados MEDLINE que teve por objetivo identificar os grupos de pesquisa brasileiros interessados no tratamento farmacológico do alcoolismo. Foi realizada busca de todos os artigos publicados a partir de 1996 com a seguinte combinação de termos: alcoholism, drug therapy, humans e Brazil. Nove artigos foram localizados, sendo sete ensaios clínicos, uma revisão e um relato de caso. Esses estudos foram conduzidos em quatro instituições diferentes, sendo que uma foi responsável por mais da metade dos artigos. Considerando as publicações no MEDLINE sobre quimioterapia do alcoolismo em humanos, esses artigos representariam $0,71 \%$ da produção científica mundial no mesmo período. Os estudos envolvem cinco fármacos, sendo três aprovados para tratamento do alcoolismo - dissulfiram, naltrexona e acamprosato; e dois em avaliação - topiramato e gabapentina. Conclui-se que os pesquisadores brasileiros estão contribuindo efetivamente para a análise do arsenal terapêutico do tratamento do alcoolismo, assim como sua expansão com novos fármacos. Entretanto, o número de grupos de pesquisa nacionais interessados por esta questão ainda é muito pequeno para a extensão do problema no País.
\end{abstract}

Palavras-chave: alcoolismo/quimioterapia; ensaio clínico; Brasil.

\begin{abstract}
Recent statistics show that millions of Brazilians are alcohol dependents. One of the main strategies for the treatment of such dependents is pharmacological therapy, which presents low success rate. In such context, it is essential that national research groups lead analyses of existing and new drugs for the treatment of alcohol dependents. This study is a narrative and critical bibliographic review of MEDLINE database aiming to identify Brazilian research groups interested in pharmacological treatment of alcoholism. A search of all articles published since 1996 was performed with the following combinations of terms: alcoholism, drug therapy, humans and Brazil. Nine articles were found, including seven clinical trials, one review and one case report. Such studies were conducted by four distinct institutions, one of them being responsible for more than half of the papers. Considering MEDLINE publications on drug therapy of alcoholism in humans, these papers represent $0.71 \%$ of the worldwide scientific production during the same period. The studies comprise five substances, three approved for alcoholism treatment - disulfiram, naltrexone, and acamprosate; - and two under evaluation - topiramate and gabapentin. It is concluded that Brazilian scientists are effectively contributing to the analysis of the therapeutical arsenal for alcoholism treatment, as well as its expansion with new medications. However, the number of national research groups interested in such question is too small considering the extension of the problem in the country.
\end{abstract}

Keywords: alcoholism/drug therapy; clinical trial; Brazil. 


\section{INTRODUCุÃO}

O álcool tem grande impacto sobre a saúde pública em decorrência do potencial de abuso. Nas sociedades ocidentais, estima-se que $90 \%$ das pessoas consomem álcool, dentre as quais $10 \%$ podem desenvolver dependência ${ }^{1}$. No Brasil, há uma estimativa de que aproximadamente $11,2 \%$ da população é dependente de álcool, o que representa mais de cinco milhões de pessoas ${ }^{2}$.

Um grande obstáculo para restrição do uso de álcool é a dificuldade de tratar o dependente. Quando estes indivíduos deixam de consumir álcool, desenvolvem uma síndrome de abstinência com sintomas altamente desagradáveis ${ }^{3}$. Por causa síndrome de abstinência, a aderência ao tratamento do alcoólatra é muito baixa.

Outra dificuldade é a falha do tratamento. Cerca de metade dos pacientes dependentes de álcool recaem após três meses de tratamento ${ }^{4}$.

Esse panorama é mundial e deixa clara a necessidade do aprimoramento da terapêutica do alcoolismo. Um das abordagens mais importantes neste sentido é a avaliação dos fármacos em uso, bem como a pesquisa de novos fármacos que possam aumentar a eficácia do tratamento. Em consequência disso, seria possível reduzir os gastos com o tratamento e poderiam ser mais bem direcionadas essas verbas para outros setores emergentes da saúde, e também sociais, na melhor reinserção do dependente ao convívio com outras pessoas.

No Brasil, a importância do álcool na saúde pública não é menor que no resto do mundo. Nesse sentido, este trabalho teve por objetivo identificar grupos de pesquisa brasileiros que estejam interessados em contribuir para a avaliação de fármacos para a terapia do alcoolismo em humanos por meio do levantamento de pesquisas publicadas em base de dados científicos internacionais.

\section{MÉTODO}

Este estudo é uma revisão narrativa crítica da literatura. A pesquisa bibliográfica foi realizada na base de dados MEDLINE utilizando a ferramenta de busca disponível em http://www.ncbi.nlm.nih.gov/pubmed. Foram pesquisados estudos clínicos publicados a partir de 1996 que tenham sido realizados no Brasil com a intenção de testar a eficácia de fármacos para o tratamento do alcoolismo em seres humanos. Para tanto, foi utilizada como estratégia de busca uma combinação da expressão alcoholism/drug therapy e os termos humans e Brazil. O termo humans foi incluído na estratégia para que não fossem selecionados artigos descrevendo estudos realizados exclusivamente em animais de experimentação.

O número de citações feitas aos artigos foi obtido da base de dados SCOPUS, disponível em http://www.scopus.com. Dados sobre a qualidade de periódicos e sobre a produtividade científica brasileira e mundial foram consultados na base de dados SCImago Journal \& Country Rank, disponível em http://www.scimagojr.com.

\section{RESULTADOS E DISCUSSÃO}

\section{Panorama geral das publicações brasileiras sobre tratamento do alcoolismo}

Nove artigos sobre estudos clínicos de fármacos para o tratamento de alcoolismo foram publicados por grupos brasileiros em revistas indexadas no MEDLINE desde 1996 (Tabela 1). Analisando o tipo de estudo, sete artigos descrevem ensaios clínicos ${ }^{5-11}$, um trata-se de um relato de caso ${ }^{12}$ e o artigo restante é uma revisão bibliográfica ${ }^{13}$. A realização de busca equivalente na base LILACS não resultou na identificação de outros artigos com estudos semelhantes que pudessem ser incluídos nesta lista.

Todos os artigos apresentam como afiliação acadêmica principal universidade ou centro de pesquisa sediado na região Sudeste (Tabela 1). O centro de pesquisa mais ativo foi o GREA (Grupo Interdisciplinar de Estudos de Álcool e Drogas) do Instituto de Psiquiatria da Universidade de São Paulo (USP), com cinco artigos; seguido pela UNIAD (Unidade de Pesquisa em Álcool e Drogas), entidade liderada por pesquisadores do Departamento de Psiquiatria da Universidade Federal da São Paulo (UNIFESP), que foi responsável por três artigos. Dois outros grupos de pesquisa participam da lista: o PROJAD (Programa de Estudos e Assistência ao Uso Indevido de Drogas) do Instituto de Psiquiatria da Universidade Federal do Rio de Janeiro (UFRJ) e o Laboratório de Ciências Cognitivas e Neuropsicofarmacologia da Universidade Federal do Espírito Santo (UFES).

Assim, apenas quatro grupos de pesquisa diferentes respondem por toda a produção científica brasileira no tratamento de alcoólatras em periódicos indexados no MEDLINE (Tabela 1). Destes, três conduziram ensaios clínicos $\left(\mathrm{GREA}^{7,8,10,11}\right.$ UNIAD $^{5,6}$ e $\mathrm{UFES}^{9}$ ). O quarto grupo (PROJAD) publicou um relato de caso $^{12}$. A única revisão narrativa ${ }^{13}$ apresenta afiliação dos dois centros mais produtivos (GREA e a UNIAD), trazendo para a literatura a experiência dos principais grupos de pesquisa brasileiros nesse campo.

Com relação aos fármacos avaliados, cada estudo avaliou um ou no máximo dois, totalizando cinco diferentes substâncias: dissulfiram, acamprosato, naltrexona, topiramato e gabapentina (Tabela 1). Entre estes, três (dissulfiram, acamprosato e naltrexona) já se encontram aprovados para uso clínico no tratamento do alcoolismo e estão entre os mais prescritos mundialmente para esse tipo de terapia ${ }^{14}$, uma vez que apresentam os melhores resultados clínicos até o presente ${ }^{15}$. Os outros dois fármacos (topiramato e gabapentina) passaram a ser testados no tratamento do alcoolismo mais recentemente e ainda estão aguardando aprovação para tal finalidade.

O topiramato é um fármaco com mecanismo de ação complexo que apresenta reconhecida efetividade clínica na epilepsia e enxaqueca, tendo sido estudado também para o tratamento de transtornos relacionados à ansiedade e à compulsividade e abuso de drogas ${ }^{16}$. Tem sido considerado como a mais promissora entre as novas alternativas farmacológicas para o alcoolismo apesar 
Tabela 1: Estudos de avaliação de fármacos para o tratamento do alcoolismo em humanos realizados por grupos de pesquisa brasileiros publicados em revistas indexadas no PubMed a partir de 1996

\begin{tabular}{|c|c|c|c|c|c|c|}
\hline Ano & Artigo & Afiliação* & $\begin{array}{l}\text { Tipo de estudo/ } \\
\text { delineamento } \\
\text { epidemiológico }\end{array}$ & $\begin{array}{l}\text { Fármacos } \\
\text { estudados }\end{array}$ & Citações** & Ref. \\
\hline 2011 & $\begin{array}{l}\text { Elbreder MF, et al. Alcohol dependence: analysis of factors associated with } \\
\text { retention of patients in outpatient treatment. } \\
\text { Alcohol Alcohol. 2011;46(1):74-6. }\end{array}$ & $\begin{array}{l}\text { UNIAD } \\
\text { São Paulo, SP }\end{array}$ & Transversal & dissulfiram & 1 & 5 \\
\hline 2010 & $\begin{array}{l}\text { Elbreder MF, et al. The use of disulfiram for alcohol-dependent patients and } \\
\text { duration of outpatient treatment. } \\
\text { Eur Arch Psychiatry Clin Neurosci. 2010;26:191-5. }\end{array}$ & $\begin{array}{l}\text { UNIAD } \\
\text { São Paulo, SP }\end{array}$ & Transversal & dissulfiram & 2 & 6 \\
\hline 2009 & $\begin{array}{l}\text { Baltieri DA, et al. The role of alcoholic beverage preference in severity } \\
\text { of alcohol dependence and adherence to the treatment. Alcohol. } \\
2009 ; 43(3): 185-95 \text {. }\end{array}$ & $\begin{array}{l}\text { GREA-USP } \\
\text { São Paulo, SP }\end{array}$ & $\begin{array}{l}\text { Ensaio clínico } \\
\text { randomizado }\end{array}$ & $\begin{array}{l}\text { topiramato e } \\
\text { naltrexona }\end{array}$ & 4 & 7 \\
\hline 2008 & $\begin{array}{l}\text { Baltieri DA, et al. Comparing topiramate with naltrexone in the treatment of } \\
\text { alcohol dependence. Addiction. 2008;103(12):2035-44. }\end{array}$ & $\begin{array}{l}\text { GREA-USP } \\
\text { São Paulo, SP }\end{array}$ & $\begin{array}{l}\text { Ensaio clínico } \\
\text { randomizado }\end{array}$ & $\begin{array}{l}\text { topiramato e } \\
\text { naltrexona }\end{array}$ & 29 & 8 \\
\hline 2008 & $\begin{array}{l}\text { Cruz M, et al. Topiramate may modulate alcohol abuse but not other } \\
\text { compulsive behaviors in frontotemporal dementia: case report. Cogn Behav } \\
\text { Neurol. 2008;21(2):104-6. }\end{array}$ & $\begin{array}{l}\text { PROJAD-UFRJ } \\
\text { Rio de Janeiro, RJ }\end{array}$ & Relato de caso & topiramato & 8 & 12 \\
\hline 2007 & $\begin{array}{l}\text { Furieri FA, Nakamura-Palacios EM. Gabapentin reduces alcohol } \\
\text { consumption and craving: a randomized, double-blind, placebo-controlled } \\
\text { trial.J Clin Psychiatry. 2007;68(11):1691-700 }\end{array}$ & UFES, Vitória, ES & $\begin{array}{l}\text { Ensaio clínico } \\
\text { randomizado }\end{array}$ & gabapentina & 24 & 9 \\
\hline 2005 & $\begin{array}{l}\text { Castro LA, Baltieri DA. The pharmacologic treatment of the alcohol } \\
\text { dependence. Rev Bras Psiquiatr. 2004;26(Suppl 1):S43-6. }\end{array}$ & $\begin{array}{l}\text { GREA-USP e UNIAD } \\
\text { São Paulo, SP }\end{array}$ & Revisão & - & 8 & 13 \\
\hline 2004 & $\begin{array}{l}\text { Baltieri DA, Andrade AG. Acamprosate in alcohol dependence: a randomized } \\
\text { controlled efficacy study in a standard clinical setting. J Stud Alcohol. } \\
\text { 2004;65(1):136-9. }\end{array}$ & $\begin{array}{l}\text { GREA-USP } \\
\text { São Paulo, SP }\end{array}$ & $\begin{array}{l}\text { Ensaio clínico } \\
\text { randomizado }\end{array}$ & acamprosato & 19 & 11 \\
\hline 2003 & $\begin{array}{l}\text { Baltieri DA, Andrade AG. Efficacy of acamprosate in the treatment of } \\
\text { alcohol-dependent outpatients. Rev Bras Psiquiatr. 2003;25(3):156-9. }\end{array}$ & $\begin{array}{l}\text { GREA-USP } \\
\text { São Paulo, SP }\end{array}$ & $\begin{array}{l}\text { Ensaio clínico } \\
\text { randomizado }\end{array}$ & acamprosato & 14 & 10 \\
\hline
\end{tabular}

de ainda ser necessário determinar a dose terapêutica e o tempo apropriado de tratamento ${ }^{17}$. A gabapentina é um anticonvulsivante que interfere na neurotransmissão mediada por GABA que tem sido usado também no tratamento de dores de origem nervosa. Os resultados do tratamento de dependentes de álcool com gabapentina são animadores, mostrando redução dos sintomas da dependência do álcool ${ }^{18}$.

Os nove artigos foram publicados em oito periódicos diferentes, todos bem conceituados. Considerando-se o parâmetro de prestígio científico SJR da base de dados SCImago Journal \& Country Rank, os periódicos encontram-se classificados entre as posições 471 (Journal of Clinical Psychiatry) e 1.724 (Revista Brasileira de Psiquiatria) entre todas as 5.304 revistas cadastradas na área de Medicina (dados não apresentados). Não foi possível realizar a comparação em categorias mais restritas (por exemplo, Psiquiatria, Toxicologia ou Neurologia Clínica), uma vez que nenhuma destas incluía todos ou a maioria desses oito periódicos.

Uma medida do impacto desses artigos na comunidade científica pode ser inferida pelo número de citações recebidas. De acordo com a base de dados SCOPUS, todos esses artigos foram citados por outros ao menos uma vez (Tabela 1). Como esperado, artigos publicados mais recentemente apresentam número menor de citações. Dois artigos chamam a atenção: Baltieri e colaboradores publicado em $2008^{8}$, com 29 citações, e Furieri e colaboradores publicado em $2007^{9}$, com 24 citações.

Para quantificar a representatividade desta contribuição para o estudo em humanos da quimioterapia do alcoolismo, a busca no MEDLINE foi realizada excluindo-se o termo Brazil e
Tabela 2: Representatividade da produção científica de grupos de pesquisa brasileiros na avaliação de fármacos, estimada a partir de busca na base de dados PubMed (1996 até o presente)

\begin{tabular}{|c|c|c|c|}
\hline \multirow{2}{*}{$\begin{array}{l}\text { Doença } \\
\text { (estratégia de busca) }\end{array}$} & \multicolumn{2}{|c|}{$\begin{array}{c}\text { Número de artigos } \\
\text { no MEDLINE }\end{array}$} & \multirow{2}{*}{$\begin{array}{l}\text { Representatividade } \\
\text { da produção } \\
\text { científica brasileira }\end{array}$} \\
\hline & Total & Brasil $^{* *}$ & \\
\hline $\begin{array}{l}\text { Alcoolismo } \\
\text { (alcoholism/drug therapy humans) }\end{array}$ & 1.267 & 9 & $0,71 \%$ \\
\hline $\begin{array}{l}\text { Diabetes } \\
\text { (diabetes/drug therapy humans) }\end{array}$ & 26.469 & 164 & $0,62 \%$ \\
\hline $\begin{array}{l}\text { Câncer } \\
\text { (cancer/drug therapy humans) }\end{array}$ & 186.726 & 928 & $0,50 \%$ \\
\hline
\end{tabular}

*Busca realizada em 4 de junho de 2012.

**0 termo Brazil foi incluído na busca.

mantendo-se a mesma restrição de período da busca inicial. Considerando-se arbitrariamente o número total de artigos encontrados (1.267) como a produção científica mundial na área desde 1996, seria possível estimar a participação brasileira como 0,71\% (Tabela 2). Essa estimativa deve se aproximar muito da proporção real. O MEDLINE é a base de dados internacional mais abrangente nas Ciências da Saúde, portanto a fonte individual de informações mais adequada a essa finalidade. Além disso, como a contagem das produções científicas mundial e brasileira foram realizadas exatamente da mesma forma, eventuais erros nos números de artigos localizados deveriam ocorrer proporcionalmente nos dois casos.

A participação brasileira de $0,71 \%$ do total de publicações sobre o tratamento de dependentes de álcool está aquém do resultado global da Ciência brasileira. Segundo dados do SCImago Journal \& Country Rank correspondentes a período equivalente da busca realizada (1996-2010), universidades e institutos de 
pesquisa brasileiros publicaram $1,47 \%$ de todos os artigos publicados no mundo, sendo 1,51\% especificamente na área Medicina (Tabela 3). Observa-se ainda uma tendência de ampliação dessa participação brasileira, que pode ser detectada quando se considera apenas o ano de 2010, havendo aumento da fração de artigos brasileiros quando se considera todas as áreas do conhecimento $(1,88 \%)$ e mais destacadamente na Medicina (2,17\%) e avanço na classificação entre todos os países (Tabela 3). Essa menor produtividade possivelmente pode estar relacionada em grande parte à dificuldade de realização de ensaios clínicos com fármacos no País. Aplicando a estratégia de busca no MEDLINE descrita a outras doenças, encontraram-se proporções da mesma ordem de grandeza para a participação brasileira em publicações mundiais: $0,62 \%$ para diabetes e $0,50 \%$ para câncer (Tabela 2 ).

\section{Avanço do conhecimento em razão de estudos clínicos brasileiros}

Uma vez caracterizados quem são os grupos de pesquisa brasileiros ativos na pesquisa do tratamento da dependência do álcool e qual sua participação na comunidade científica internacional, seus ensaios clínicos publicados são discutidos a seguir, organizados por fármaco(s) analisado(s).

\section{Dissulfiram}

Pesquisadores do UNIAD realizaram estudo transversal de mais 800 pacientes no período 2000-2006 sobre o uso do dissulfiram que originou duas publicações ${ }^{5,6}$. O estudo demonstrou que os pacientes que permaneceram em tratamento durante o primeiro ano completo usaram proporcionalmente mais dissulfiram do que aqueles que permaneceram por período de tratamento mais curto. Além disso, a permanência após quatro semanas de tratamento ambulatorial da dependência do álcool era maior entre aqueles pacientes usando alguma medicação, principalmente o dissulfiram. Os autores concluem que o dissulfiram, apesar de ser uma medicação antiga, pode ser útil no tratamento do alcoolismo.

\section{Topiramato e naltrexona}

Em estudo do GREA ${ }^{8}$, comparou-se a eficácia do topiramato em relação à naltrexona no tratamento da dependência por álcool em ensaio duplo-cego, controlado por placebo envolvendo 155 pacientes. Após uma semana de detoxificação, os pacientes foram aleatoriamente distribuídos em grupos de tratamento com topiramato, com naltrexona ou com placebo. A análise estatística dos dados coletados mostrou que o topiramato foi superior ao placebo em relação a: (1) tempo para primeira recaída; (2) período de abstinência; e (3) porcentagem de sujeitos em abstinência após quatro e oito semanas.

Esses resultados permaneceram significativos mesmo após consideração do efeito de participação dos pacientes em sessões dos Alcoólicos Anônimos, que era maior no grupo de topiramato em relação aos demais. Os pesquisadores não detectaram diferenças estatísticas entre o grupo da naltrexona em relação ao placebo e da naltrexona em relação ao topiramato, mas o grupo topiramato apresentou uma tendência de maior eficácia na prevenção da recaída ao alcoolismo em relação à naltrexona. Um segundo artigo derivado do mesmo ensaio clínico ${ }^{7}$ ressaltou que uma variável relevante para o resultado do tratamento seria o tipo de bebida alcoólica consumida. Bebedores preferenciais de cerveja apresentaram maior aderência ao tratamento que bebedores de destilados, independentemente da medicação usada.

\section{Gabapentina}

Pesquisadores da Universidade Federal do Espírito Santo examinaram a eficácia da gabapentina no tratamento da síndrome de dependência e de abstinência tardia ao álcool, como também os efeitos desse tratamento em vários testes cognitivos que avaliam a atenção, a memória de curto prazo e as funções executivas em alcoolistas 9 . Foi realizado um ensaio clínico randomizado, duplo-cego, conduzido em um serviço municipal especializado com uma amostra de 60 pacientes dependentes do álcool do sexo masculino sem complicações clínicas ou psiquiátricas. Após seleção e tratamento da síndrome de abstinência aguda por pelo menos sete dias, 30 pacientes receberam gabapentina e outros 30 receberam placebo durante quatro semanas. Após os 28 dias de tratamento, o grupo gabapentina reduziu significativamente o número de doses por dia, as doses por consumo e a porcentagem de beber pesado, e aumentou a porcentagem de dias em abstinência quando comparados ao placebo. O comportamento obsessivo-compulsivo diminuiu significativamente em ambos os grupos, sendo mais significativo para o grupo gabapentina para o automatismo. A insônia se apresentou em ambos os grupos, porém o grupo gabapentina usou menor quantidade de benzodiazepínicos. O desempenho cognitivo foi bastante prejudicado em todos os testes em ambos os grupos. No entanto, a gabapentina produziu uma melhora maior no processamento da atenção

Tabela 3: Representatividade da produção científica brasileira de acordo com a base de dados SCImago Journal \& Country Rank

\begin{tabular}{|c|c|c|c|c|c|}
\hline \multirow{2}{*}{ Período } & \multirow{2}{*}{ Área do conhecimento } & \multicolumn{2}{|c|}{ Número de documentos } & \multicolumn{2}{|c|}{$\begin{array}{l}\text { Representatividade da produção } \\
\text { científica brasileira }\end{array}$} \\
\hline & & Todos os países & Brasil & Porcentagem (\%) & Ranking \\
\hline \multirow{2}{*}{ 1996-2010 } & Todas & 22.395 .845 & 328.361 & 1,47 & $15^{\circ}$ \\
\hline & Medicina & 5.395 .763 & 81.230 & 1,51 & $16^{\circ}$ \\
\hline \multirow{2}{*}{2010} & Todas & 2.406 .772 & 45.189 & 1,88 & $13^{\circ}$ \\
\hline & Medicina & 551.691 & 11.957 & 2,17 & $13^{\circ}$ \\
\hline
\end{tabular}


verbal e das memórias verbal e visuoespacial. Os autores concluíram que a gabapentina reduziu o consumo de álcool e diminuiu a compulsão do beber, favorecendo a permanência da abstinência e a melhora de algumas funções cognitivas. Esses resultados, aliados à ausência de efeitos colaterais e à segurança do fármaco, podem sugerir a possibilidade de seu uso no tratamento na síndrome de dependência do álcool.

\section{Acamprosato}

Outras pesquisas desenvolvidas pelo GREA da Universidade de São Paulo ${ }^{10,11}$ estudaram a eficácia e a segurança do acamprosato no tratamento ambulatorial de 75 pacientes do sexo masculino com diagnóstico de dependência de álcool por meio de estudo duplo-cego controlado e randomizado. Após um período de desintoxicação de uma semana, os pacientes foram divididos aleatoriamente em dois grupos: o primeiro grupo recebeu acamprosato por 12 semanas, e o segundo, placebo pelo mesmo período de tempo. Em seguida, todos continuaram o tratamento por mais 12 semanas sem uso de medicação. Na última semana do tratamento, $42,5 \%$ dos pacientes do grupo tratado por acamprosato permaneciam em abstinência desde a primeira consulta, enquanto para o grupo controle isso foi observado em apenas $20 \%$ dos pacientes. Vinte e cinco por cento dos pacientes que estavam recebendo acamprosato e $20 \%$ dos pacientes que estavam recebendo placebo abandonaram o tratamento. Poucos efeitos colaterais foram registrados em ambos os grupos. O acamprosato mostrou ser seguro e eficaz no tratamento de pacientes dependentes de álcool e na manutenção da abstinência durante 24 semanas.

\section{CONSIDERAÇÕES FINAIS}

A pesquisa bibliográfica de ensaios clínicos brasileiros para o tratamento farmacológico da dependência do álcool mostrou que cinco dos mais importantes fármacos dessa classe já foram avaliados por grupos de pesquisa nacionais, sendo três aprovados para esse uso - dissulfiram, naltrexona e acamprosato - e dois em avaliação - topiramato e gabapentina. Todos os artigos publicados receberam citações, mostrando sua penetração na comunidade científica específica. Conclui-se que os pesquisadores brasileiros estão contribuindo efetivamente tanto para a avaliação do arsenal terapêutico disponível para o tratamento do alcoolismo como para sua ampliação com novos fármacos. Destacase o estudo brasileiro ${ }^{9}$ sobre a gabapentina, uma vez que ainda são poucos os artigos sobre a aplicação desse fármaco na dependência do álcool ${ }^{18}$. Por outro lado, não se encontrou artigos sobre estudos brasileiros com outros fármacos de interesse no tratamento do alcoolismo, como baclofen e ondansentron ${ }^{17}$.

Foram localizados apenas quatro grupos de pesquisa nacionais que conseguiram publicar seus estudos em periódicos científicos indexados na principal base de dados internacional para as Ciências da Saúde. Além de serem poucos, esses grupos estão localizados exclusivamente na região Sudeste do País.

As publicações brasileiras no tratamento do alcoolismo representam $0,71 \%$ da produção científica mundial neste campo, abaixo da participação brasileira na Medicina em geral no mesmo período (1,51\%). Assim, acredita-se que seria desejável ao menos dobrar a produção científica nessa área. Para tanto, certamente seria necessário aumentar o número de grupos de pesquisa e idealmente distribuí-los mais uniformemente pelo País. Aparentemente, também há necessidade de se melhorar as condições para realização de ensaios clínicos no Brasil, uma vez que produtividade de publicação inferior à média nacional também foi detectada para os grupos de pesquisa interessados no tratamento farmacológico de outras doenças (diabetes e câncer).

Assim, a análise da pesquisa brasileira sobre o tratamento da dependência do álcool resultou em poucos artigos de boa qualidade produzidos por escassos grupos de pesquisa. Essa produção é insatisfatória especialmente considerando-se a dimensão do problema no País. Várias são as razões para que a comunidade científica brasileira produza mais pesquisa de alto nível na farmacologia do alcoolismo: formação de pessoal qualificado com capacidade para conduzir e discutir esses tratamentos; adaptação de protocolos internacionais às necessidades de nossa população e nossa infraestrutura de saúde; proposta de novos esquemas terapêuticos e novos fármacos. Sugere-se que as agências de fomento à pesquisa e órgãos governamentais estimulem ainda mais a pesquisa nessa área e que as universidades e institutos de pesquisa respondam com pujança a esta chamada.

\section{REFERÊNCIAS}

1. Baltieri DA. Tratamento farmacológico do alcoolismo. São Paulo: Lemos Editorial; 2005. p.17-61.

2. Carlini EA, Galduróz JCF, Noto AR, Nappo SA. Levantamento domiciliar de drogas psicotrópicas no Brasil: estudo envolvendo as 107 maiores cidades do país - 2001. São Paulo: CEBRID/ UNIFESP: 2001.

3. Laranjeira R, Nicastri S, Jeronimo C, Marques AC. Consenso sobre a Síndrome de Abstinência do Álcool (SAA) e o seu tratamento. Rev Bras Psiquiatr. 2000;22(2):62-71
4. Monras M, Freixa N, Ortega L, Lligoña A, Mondón S, Gual A. Efficacy of group therapy of alcoholics. Results of a controlled clinical trial. Med Clin (Barc). 2000;115(4):126-31.

5. Elbreder MF, Silva RS, Pillon SC, Laranjeira R. Alcohol dependence: analysis of factors associated with retention of patients in outpatient treatment. Alcohol Alcohol. 2011;46(1):74-6.

6. Elbreder MF, de Humerez DC, Laranjeira R. The use of disulfiram for alcohol-dependent patients and duration of outpatient treatment. Eur Arch Psychiatry Clin Neurosci. 2010;260(3):191-5. 
7. Baltieri DA, Daró FR, Ribeiro PL, Andrade AG. The role of alcoholic beverage preference in severity of alcohol dependence and adherence to the treatment. Alcohol. 2009;43(3):185-95.

8. Baltieri DA, Daró FR, Ribeiro PL, Andrade AG. Comparing topiramate with naltrexone in the treatment of alcohol dependence. Addiction. 2008:103(12):2035-44.

9. Furieri FA, Nakamura-Palacios EM. Gabapentin reduces alcohol consumption and craving: a randomized, double-blind, placebocontrolled trial.J Clin Psychiatry. 2007;68(11):1691-700.

10. Baltieri DA, Andrade AG. Efficacy of acamprosate in the treatment of alcohol-dependent outpatients. Rev Bras Psiquiatr. 2003;25(3):156-9.

11. Baltieri DA, Andrade AG. Acamprosate in alcohol dependence: a randomized controlled efficacy study in a standard clinical setting. $J$ Stud Alcohol. 2004:65(1):136-9.

12. Cruz M, Marinho V, Fontenelle LF, Engelhardt E, Laks J. Topiramate may modulate alcohol abuse but not other compulsive behaviors in frontotemporal dementia: case report. Cogn Behav Neurol. 2008;21(2):104-6.

13. Castro LA, Baltieri DA. The pharmacologic treatment of the alcohol dependence. Rev Bras Psiquiatr. 2004;26(Suppl 1):S43-6.
14. Jupp B, Lawrence AJ. New horizons for therapeutics in drug and alcohol abuse. Pharmacol Ther. 2010;125(1):138-68.

15. Rueff B. Evaluation of drug treatment of primary alcoholism. Rev Prat 1999;49(4):400-2.

16. Johnson BA, Ait-Daoud N. Topiramate in the new generation of drugs: efficacy in the treatment of alcoholic patients. Curr Pharm Des. 2010;16(19):2103-12.

17. Edwards S, Kenna GA, Swift RM, Leggio L. Current and promising pharmacotherapies, and novel research target areas in the treatment of alcohol dependence: a review. Curr Pharm Des. 2011; 17(14):1323-32

18. Clemens KJ, Vendruscolo LF. Anxious to drink: gabapentin normalizes GABAergic transmission in the central amygdala and reduces symptoms of ethanol dependence. J Neurosci. 2008;10; 28(37):9087-9.

Endereço para correspondência

Ricardo Peres do Souto - Avenida Príncipe de Gales, 821 CEP 09060-650 - Santo André (SP), Brasil -

E-mail: ricardo.souto@fmabc.br

Conflito de interesse: nada a declarar. 